\title{
Finding a Walrasian Equilibrium is Easy for a Fixed Number of Agents
}

\author{
FEDERICO ECHENIQUE, Humanities and Social Sciences, California Institute of Technology \\ ADAM WIERMAN, Computing and Mathematical Sciences, California Institute of Technology
}

\begin{abstract}
The problem of computing Walrasian equilibria has been studied continually over many decades, and the basic message that has emerged from the literature is negative: computing a Walrasian equilibrium tends to be "hard" in general settings. For example, finding a Walrasian equilibrium is hard (PPAD-complete and hard to approximate) even in economies with piece-wise linear concave utilities that are separable. Similarly,
\end{abstract} the problem is also hard in economies in which agents have Leontief preferences.

In contrast to such negative results, in this paper we obtain a positive result for a restricted, but important, setting: the setting with a fixed the number of agents. Specifically, we exhibit an algorithm for finding an approximate Walrasian equilibrium under general, but well-behaved, utility functions, that runs in polynomial-time in the number of goods, when the number of agents is fixed.

Our algorithm is based on the Negishi approach for proving existence of Walrasian equilibria, which uses the second welfare theorem to translate the problem of finding a Walrasian equilibrium into a fixed point calculation in the space of welfare weights. Welfare weights have dimensionality dependent on the number of agents in the market. We show that, for the purposes of approximating equilibrium, the space of weights can be discretized in a way that is independent of the number of goods: the resulting discretization is a crucial component of our algorithm.

Our restriction to a fixed number of agents simplifies the computational problem dramatically, but still captures many applications of general equilibrium theory in economics. Specifically, most modern applications of general equilibrium theory are in the areas of macroeconomics and finance. In these settings the theory is most often applied to large economies, in the sense of having infinitely many goods, but the number of agents remains small and fixed. This is because in macroeconomic and financial applications the time horizon is usually infinite, which implies that there are infinitely many different goods.

Additionally, our result applies to some situations where there are many agents, but there is some form of limited heterogeneity. More specifically, our results apply directly to (i) economies where the behavior of many agents can be aggregated into the behavior of a single "representative consumer;" and (ii) economies with "endowment classes," where agents are fully heterogeneous in their preferences but not in their sources of income.

Finally, an important application of our results is to Fisher equilibria: we obtain existing positive results on computing Fisher equilibria as special cases of our result.

Categories and Subject Descriptors: F.2.0 [Analysis of Algorithms and Problem Complexity]: General

General Terms: Algorithms, Economics, Theory

Additional Key Words and Phrases: Computational Complexity, Walrasian Equilibria, Fisher Equilibria

This work is supported by the National Science Foundation, under grant CCF-1101470. We thank Chris Umans, Jeremy Hurwitz, Daniel Golovin, and Ilya Segal for their advice and comments on this work.

The full version of this paper is available at http://users.cms. caltech.edu/ adamw/publications-0.html

Copyright is held by the author/owner(s).

EC'12, June 4-8, 2012, Valencia, Spain.

ACM 978-1-4503-1415-2/12/06. 